\title{
APPLICATION OF ALANINE/ESR SPECTRUM SHAPE CHANGE IN GAMMA DOSIMETRY
}

\author{
HOON CHOI*, JEONG IN KIM, BYUNG ILL LEE and YOUNG KI LIM \\ Radiation Health Research Institute \\ 388-1, Ssangmoon-dong, Dobong-gu, Seoul, Korea, 132-703 \\ "Corresponding author. E-mail : 93105117@khnp.co.kr \\ Received June 19, 2009 \\ Accepted for Publication March 24, 2010
}

\begin{abstract}
Alnine pellets were installed in a nuclear power plant for one or two operation cycles and measured by electron spin resonance (ESR) spectrometers for dosimetry. Dose and "x/y ratio", i.e., satellite peak over main center peak ratio, were measured for the returned alanine dosimeters from the nuclear power plant and compared to the values of reference alanine dosimeters exposed only to gamma rays. The variation of the $\mathrm{x} / \mathrm{y}$ ratio change depended on the population of radicals from each radiation component with different LET. The gamma dose in a mixed radiation field was estimated by an additive gamma ray irradiation experiment and the measured dose rate at specified locations in the containment building.
\end{abstract}

KEYWORDS : EPR, ESR, Dosimetry, Resonance, Spectroscopy, Alanine, Radical, LET, Cable Aging

\section{INTRODCTION}

Temperature and radiation are two of the major environmental elements exerting aging effects on the incontainment instrumentation and control cables connected to major components. The effect of radiation on cable material is related to the total absorbed dose, which is mainly dependent on gamma rays [1]. For validity of radiation measurement, at least two operation cycles are required for the installation period. Generally used personal dosimeters are not appropriate for this application due to their rapid signal fading feature and relatively poor linearity at a range of high dose. Alanine-electron spin resonance (ESR) dosimetry material, meanwhile, has been proved to be suitable for long term measurement on the basis of the following factors: small signal dependency on temperature $(\fallingdotseq 0.2 \% / \mathrm{K})$, limited signal fading $(\fallingdotseq 1 \% /$ year $)$, and low influence of humidity, which can be easily overcome by hermetic sealing of capsules [2]. Based on the aforementioned advantages as a long term dosimetry material, in the present study dozens of pellet type alanine dosimeters were installed at selected positions in a nuclear power plant (NPP) for two operation cycles. ESR dosimety is known as a retrospective dosimetry method that can be applied in an emergency after an accident and is accepted as a secondary standard and transfer dosimeter for the comparison of dose quantity between laboratories [3-5]. Recently, it was found that the peaks of spectra are mainly due to three kinds of radicals induced by radiation [6-8].
The central resonance peak amplitude of main peaks is used to estimate radiation dose quantity. The weak "satellite line" beside the central resonance peak has been interpreted as the combined effects of "spin flip" of neighboring hydrogen protons of the methyl group of the paramagnetic center and a $R_{2}$ radical [9-11]. The ratio of the "satellite line and main central resonance peak" is frequently expressed as the " $\mathrm{x} / \mathrm{y}$ ratio". A decrease of this ratio has been explained by less saturation resistance of the main peak amplitude, which is caused by a decrease of the spin-lattice relaxation time $T_{l}$. Crystallographic defects and plastic deformation in the structure of the alanine lattice by thermal shock of high linear energy transfer (LET) radiation have been reported as the most likely causes of relaxation time change. The "x/y ratio" irradiated with mixed radiation, composed of two radiations with different LET values, was reported to be intermediate and is determined by the absolute population size of each radical produced by radiation with different LET values. In this paper, the " $\mathrm{x} / \mathrm{y}$ ratio" of alanine dosimeters returned from the containment building of the NPP is lower than that of dosimeters exposed to gamma rays. Furthermore, the ratio constantly decreased in proportion to the dose quantity, which corresponds with the installation period in the NPP. These results showed good accordance with previously reported results. Application of the above features to estimate gamma dose in mixed radiation fields is based on the observation that change of the " $\mathrm{x} / \mathrm{y}$ ratio" with population size of radicals is almost same at five positions in NPP. 

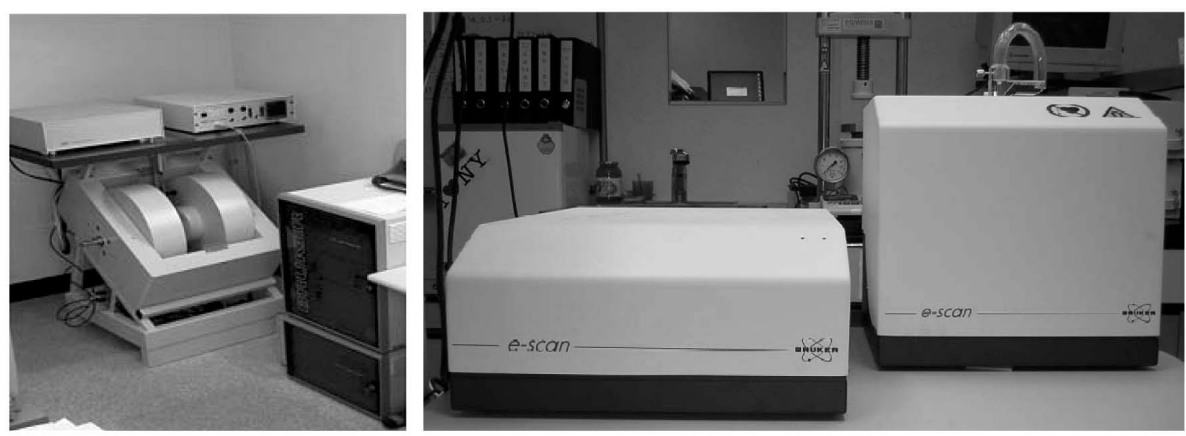

(a)
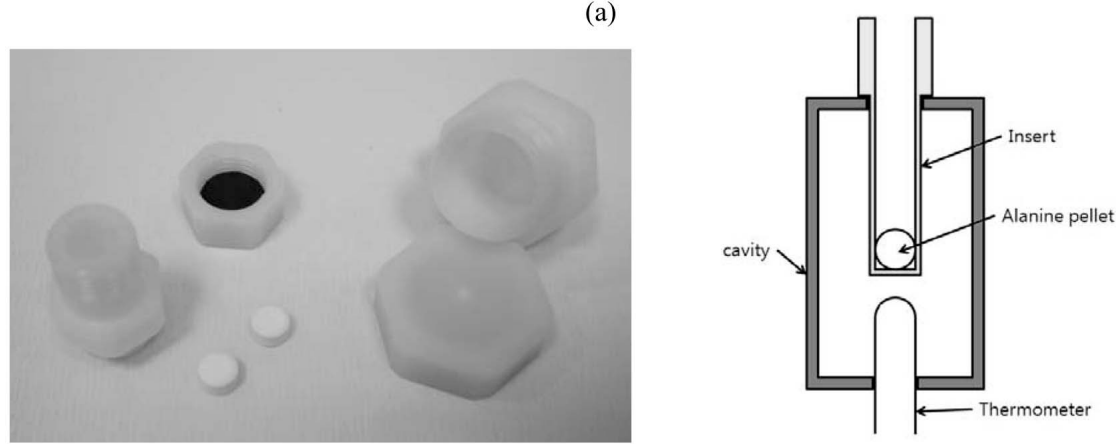

(b)

Fig. 1. (a) EMX Spectrometer (Left) and e-scan (Right) (b) Alanine Dosimeters and Capsules of Acetal (Left) and Cross Section of Alanine Dosimeter Insert of Teflon Used to Hold Dosimeter Inside EMX Spectrometer Cavity (Right)

\section{EXPERIMENT}

\subsection{Dosimeters and Spectrometry Systems}

Two ESR spectroscopy systems were used for these experiments, as shown in Fig. 1 (a). Dosimetry was performed by e-scan, which is commercially manufactured for use solely in alanine dosimetry. For the shape comparison of the spectra, EMX spectrometer was used with various different parameters. As alanine dosimeters, BioMax commercial alanine dosimeters, which contained $\alpha$ amino acid alanine, $\mathrm{CH}_{3}-\mathrm{CH}\left(\mathrm{NH}_{2}\right)-\mathrm{COOH}$, and Teflon as a binding material (ratio $>9 / 1$ ), were purchased and stored in darkness before installation. Alanine dosimeters were $5 \mathrm{~mm}$ in diameter and $3 \mathrm{~mm}$ in height and weighed about $64.5 \pm 0.5 \mathrm{mg}$. Standard alanine dosimeters for making doseresponse curves for e-scan and EMX spectrometer were irradiated in the National Physical Laboratory (NPL, United Kingdom) using ${ }^{60} \mathrm{Co}$ for various radiation ranges of gamma rays.

Standard dosimeters were surrounded by a water equivalent material and irradiated with radiation of $40 \mathrm{~Gy} / \mathrm{h}$ (absorbed dose to water was measured by standard graphite calorimeter). Temperature for irradiation was within a range of 16 to $18^{\circ} \mathrm{C}$. The other additive gamma irradiation to alanine dosimeters was done by ${ }^{137} \mathrm{Cs}$ at a dose rate of $6 \mathrm{~Gy} / \mathrm{min} \pm 4 \%$ using a blood irradiator (IBL437C, France). For estimation of uncertainties, $0.25,1$, and $3 \mathrm{kGy}$ of gamma rays were irradiated and measured. In the experiment with additive dose irradiation, 50 to 100 Gy of gamma rays were irradiated to the alanine dosimeters from the NPP.

\subsection{Dosimeter Installation and Measurement}

The installation period of alanine pellets was one or two operation cycles of "Wolsung" NPP. 30 installation positions were selected and 3 alanine dosimeters were installed at each position. The criterion for selecting positions was importance to the safety of the NPP. Capsules, containing alanine dosimeters, made of plastic (acetal) were carefully fastened to the cables to prevent shock or vibration from external force. After one operation cycle, one of the three alanine pellets was exchanged with a new pellet for measurement of changes after the first operation cycle. After the second operation cycle, all the dosimeters were collected at the time of periodic maintenance of the NPP $\left(1^{\text {st }}\right.$ operation cycle: 382 days, $2^{\text {nd }}$ operation cycle: 423 days). All the dosimeters returned from the NPP were stored under stable conditions in darkness (temperature: $21 \sim 26^{\circ} \mathrm{C}$, relative humidity: $16 \sim 22 \%$ ). For determination of the absorbed dose of alanine dosimeters, measurements were performed at least eight times on a daily basis after two or three weeks and data was corrected by the factor of the dosimeter mass and the irradiation temperature in the NPP $\left(0.14 \% / \mathrm{K}, 25^{\circ} \mathrm{C}\right)$ [12]. To accurately measure the peak to peak ratio ("x/y ratio" or No 7 over No 8 ratio in Fig. 2), environmental factors for measurement of the dose and peak ratio were controlled in the following ranges 


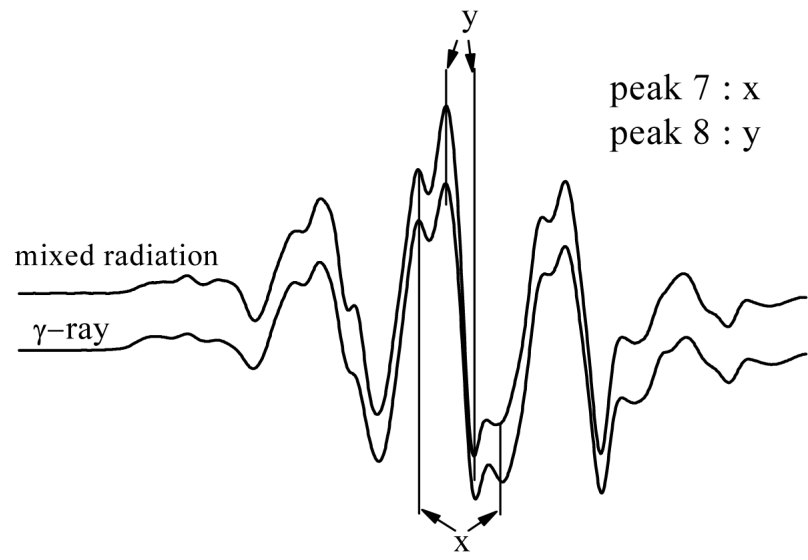

Fig. 2. Spectra Shape of Dosimeters Showed Slight Variation (a) Upper Spectra is Exposed to Mixed Radiation in the NPP (1,800Gy) (b) Lower Spectra is Exposed to Only Gamma Rays at the Laboratory $(1,500 \mathrm{~Gy})$

(temperature: $23.4 \sim 24.1^{\circ} \mathrm{C}$, relative humidity: $17 \sim 20 \%$ ). Measurement for comparison with the spectra was performed only for five positions with an EMX spectrometer with a X-band bridge and a single or rectangular dual cavity. The dual cavity was used in the case of using a standard marker for compensating $\mathrm{Q}$ factors. The " $\mathrm{x} / \mathrm{y}$ ratio" of the alanine dosimeters was highly sensitive to variation of temperature and humidity inside the cavity of the EMX spectrometer, all the data was measured on a daily basis and the data measured in the outer range of temperature and humidity limits were discarded for precise comparison. The " $\mathrm{x} / \mathrm{y}$ ratio" was also very sensitive to conditions such as the angle and position of the dosimeter inside the cavity. The $\mathrm{Q}$ value and the time for equilibrium were fixed at constant levels in the experiment. Measurement parameters were as follows: the power level of microwave and modulation amplitude was $30 \mathrm{~mW}$ and $2 \times 10^{-4} \mathrm{~T}$. This power level is much higher than used for normal measurement, because the resonance signal of alanine began to saturate at about $0.5 \mathrm{~mW}$, and did not increase linearly with the square root of increasing microwave power. This high level of microwave power is sufficient for observation of the satellite peak, which was not shown at the range of low power levels. For the purpose of accurate measurement of the " $\mathrm{x} / \mathrm{y}$ ratio", a special dosimeter holder (insert) was made for positioning the alanine pellet at exactly the same position as indicated in Fig. 1 (b). The position of the alanine dosimeter and the dosimeter holder was determined by repeated measurements for optimum conditions. All the uncertainties involved in measurement of the alanine dosimeters by spectrometers, i.e., the e-scan and EMX spectrometer, were calculated via the experiment and are listed in Table 1. Equilibrium time in the cavity before each measurement was at least 15 minutes and was maintained at a constant value in the experiment. The " $\mathrm{x} / \mathrm{y}$ ratio" was highly sensitive to
Table 1. Uncertainties of Measured Dose and " $x / y$ ratio"

\begin{tabular}{l|c|c|c}
\hline \multicolumn{1}{c|}{ Dose } & $250 \mathrm{~Gy}$ & $1,000 \mathrm{~Gy}$ & $3,000 \mathrm{~Gy}$ \\
\hline $\begin{array}{l}\text { Expanded combined } \\
\text { uncertainty } \\
\text { (e-scan spectrometer) }\end{array}$ & $5.33 \%$ & $2.97 \%$ & $2.44 \%$ \\
\hline $\begin{array}{l}\text { Response variation } \\
\text { of "x/y ratio" } \\
\text { (EMX spectrometer) }\end{array}$ & $0.91 \%$ & $0.25 \%$ & $0.16 \%$ \\
\hline $\begin{array}{l}\text { Repetition variation } \\
\text { of "x/y ratio" } \\
\text { (EMX spectrometer) }\end{array}$ & $0.80 \%$ & $0.34 \%$ & $0.12 \%$ \\
\hline
\end{tabular}

temperature inside the cavity of spectrometer, as mentioned before. The correction factor compensating the ratio change with respect to temperature at the accepted range of temperature movement was determined by use of a temperature and relative humidity meter, which was installed at the bottom of the cavity, as shown in Fig. 1 (b).

\section{RESULTS AND DISCUSSION}

The spectrums of alanine samples irradiated with only gamma rays and with both gamma rays and neutrons showed different shapes, as seen in Fig. 2.

The "x/y ratio", determined from Fig. 2, was used in this paper as an indicator of the change of the spectra shape of the alanine dosimeters. In general, the ratio of the spectra decreased when the dosimeters were exposed to additional high-LET radiation. It has been previously reported that the " $\mathrm{x} / \mathrm{y}$ ratio" showed an intermediate value in mixed radiation fields composed of high and low LET radiation [11]. In the case of alanine dosimeters returned from the NPP, most of the deposited energy likely originates from gamma rays, thermal and fast neutrons, and proton particles induced by the ${ }^{14} \mathrm{~N}(\mathrm{n}, \mathrm{p}){ }^{14} \mathrm{C}$ reaction. Change in the " $\mathrm{x} / \mathrm{y}$ ratio" could be observed from the experiment with power variation and from a comparison of peak amplitude, as seen in Fig. 3. The amplitude difference of peak 7 at microwave power level is relatively distinct while the amplitude of peak 8 is almost the same. When experiment was performed at a fixed power level of $30 \mathrm{~mW}$, as in Fig. 4, the " $\mathrm{x} / \mathrm{y}$ ratio" in the cause of exposure only to gamma rays at a range of 0.2 to $3 \mathrm{kGy}$ was constant at about 0.77 . However, for alanine dosimeters located at five positions and irradiated for one or two operation cycles in the containment building of the NPP, the " $\mathrm{x} / \mathrm{y}$ ratio" clearly decreased, in contrast to dosimeters exposed to gamma rays. At positions No 26, 35, 47, two different dosimeters were measured, as seen in Fig. 4. One is installed for the first operation cycle and the other is 


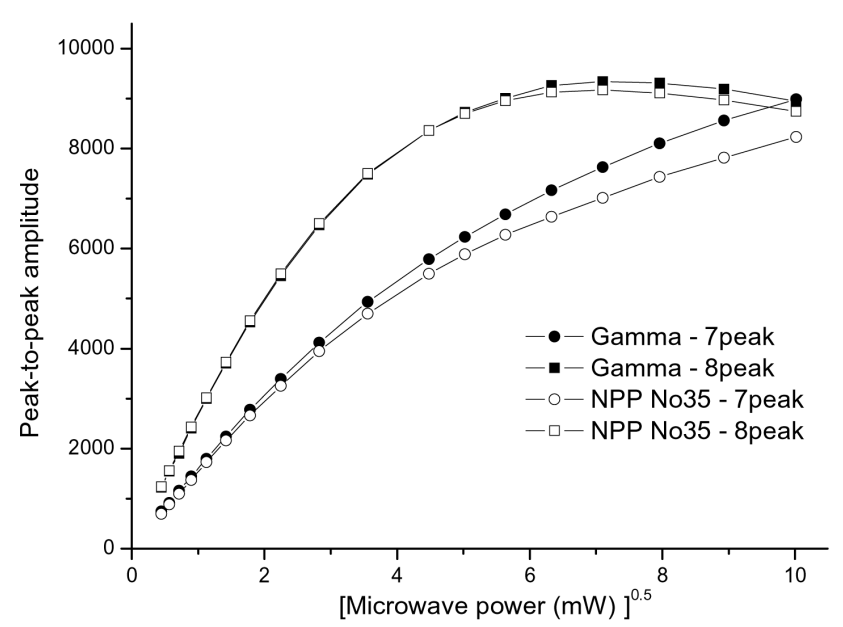

Fig. 3. Saturation Characteristics of Peak-to-peak Amplitude of Reference Gamma Exposed Alanine Dosimeter and Mixed Field Irradiated Alanine Dosimeter Returned from the NPP

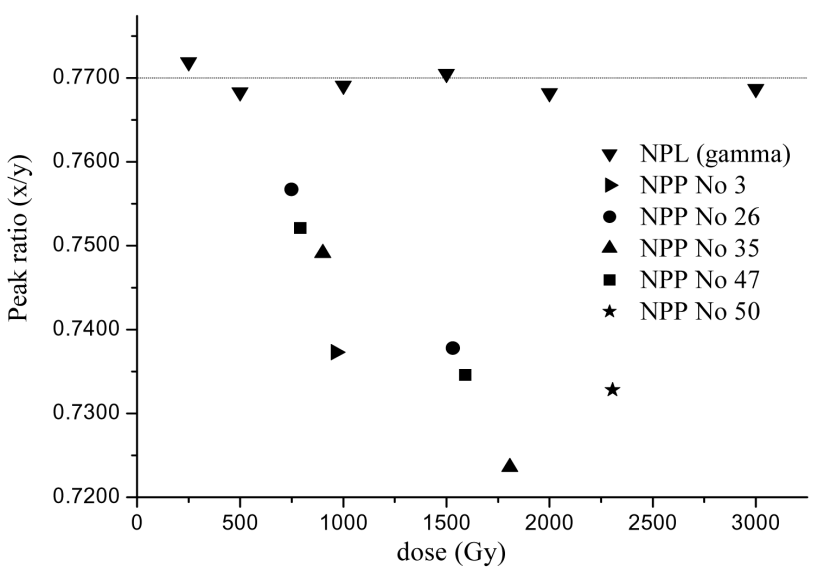

Fig. 4. "x/y ratio" of $\gamma$ Ray Irradiated from the NPL was Near 0.7700 . The Ratio of Dosimeters Returned from the NPP after One or Two Operation Cycles was much Lower than that of the Gamma Ray Exposed Dosimeters

installed for both the first and second operation cycles.

The "x/y ratio" appeared to decrease continuously and the value was inversely proportional to the radiation dose quantity. This linear decrease of the " $\mathrm{x} / \mathrm{y}$ ratio" is interesting, because the value was only dependent on the inverse of dose quantity. One possible explanation of this is that the ratio was not determined by the relative ratio of the number of radicals but by the absolute number of radicals produced by each component of mixed radiation. These results are in good agreement with previous reports that the " $\mathrm{x} / \mathrm{y}$ ratio" is changed by the absolute population size of radicals produced by each component of mixed radiation fields. In Fig. 4, the rate of change of the " $\mathrm{x} / \mathrm{y}$ ratio" versus dose quantity at No 35,47 , and 26 positions
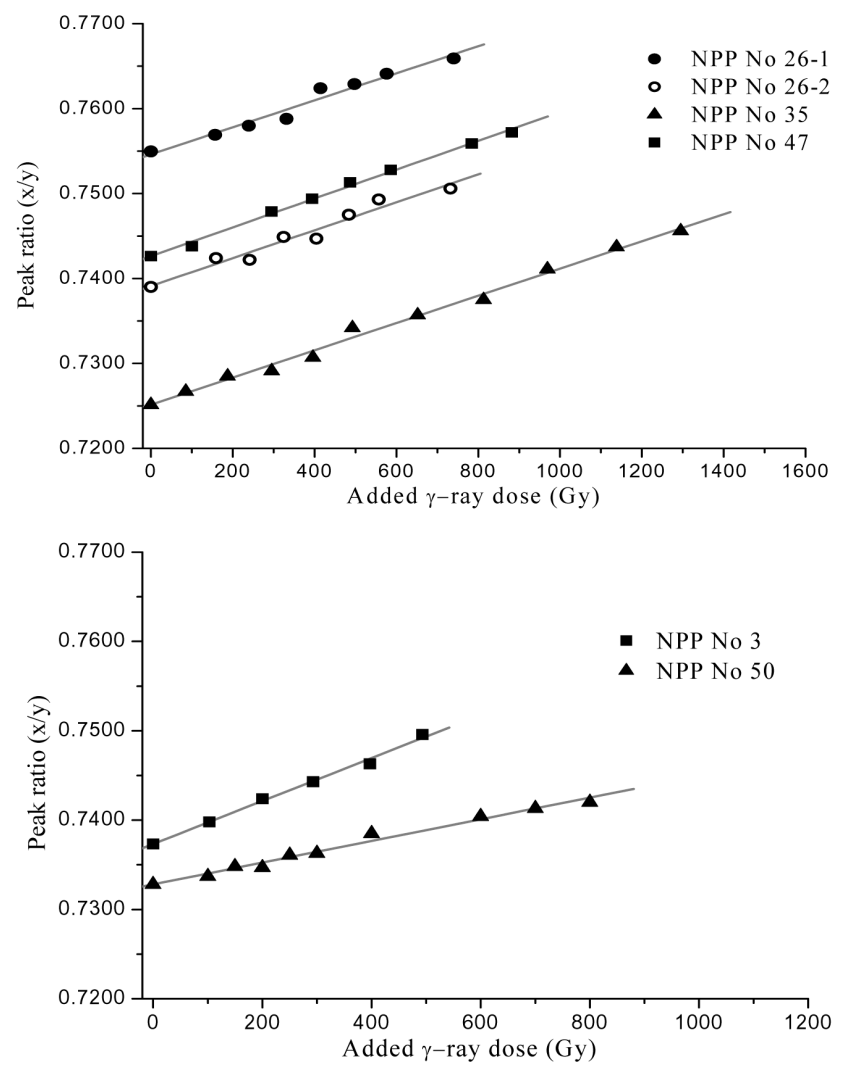

Fig. 5. "x/y ratio" Change by Additive Gamma Irradiation to the Returned Alanine Dosimeters from the NPP

for the first and second operation cycles is very similar. This means that the energy spectra of the neutrons at these positions are similar, although neutron fluence and gamma dose rate are different, because the rate of change of the " $\mathrm{x} / \mathrm{y}$ ratio" is related to the radiation quality of neutrons. The observed linear increase of the ratio indicates that the combined effect on the " $\mathrm{x} / \mathrm{y}$ ratio" by the mixed radiation field is almost balanced at a limited dose range and the effects on the " $x / y$ ratio" by each source of radiation could be estimated by additively irradiating gamma rays to the dosimeters returned from the NPP.

Although the estimation of effect by gamma rays (" $\Delta R_{\Gamma}$ ") on the " $\mathrm{x} / \mathrm{y}$ ratio" by additive gamma irradiation to the dosimeters from the NPP is not precisely equivalent to that of gamma irradiation in the mixed radiation field at the NPP, the rate of change of the " $\mathrm{x} / \mathrm{y}$ ratio" was estimated by irradiation of the additive gamma dose (about 50 to $100 \mathrm{~Gy}$ ) to the dosimeters returned from the NPP. After irradiation of additive gamma rays, as seen in Fig. 5, the " $\mathrm{x} / \mathrm{y}$ ratio" increased continuously with the dose quantity at the same rate in the case of the No 26,35 , and 47 positions.

Dosimeters at positions No 3 and 50 showed different ratios on additive gamma rays. This difference could be 
explained by the different lattice structure of alanine material and the spatial distribution of radicals compared with those of No 26, 35, and 47 dosimeters. These rates of change were used in equation (4) as parameter " $\Delta R_{\Gamma}$ ". In limited dose ranges, it appears that an increase of the ratio was caused by gamma rays and a decrease of the ratio was caused by neutrons in the mixed radiation field. As a result, the role of gamma rays in the mixed radiation field could be regarded as a component that increases the resistance of signal saturation of the main peak in the mixed radiation field. Estimation of the number of gamma ray induced radicals was attempted, on the assumption that the contribution of gamma rays to the " $\mathrm{x} / \mathrm{y}$ ratio" in the mixed radiation field in the NPP is the same as that of additive gamma irradiation. Equation (1) is generally used for dealing with a mixed radiation field. If we only consider the number of radicals in the alanine dosimeters, it can be written as:

$$
G_{c o} D=G_{\gamma} D_{\gamma}+G_{n} D_{n}
$$

As delineated in the above equation, the total number of radicals in the alanine dosimeter after irradiation by the mixed radiation field is expressed as a unit of ${ }^{60} \mathrm{Co}$ equivalent dose and $G$ value. The reason for this is that the spectrometers were calibrated by use of a standard dosimeter irradiated by gamma rays. The $G$ value denotes the radical yield of the alanine dosimeters and the subscript denotes whether the radiation source is from gamma rays or neutrons.
For equation (1), if the change of the " $\mathrm{x} / \mathrm{y}$ ratio", $\Delta R$, is multiplied, because the ratio change only depends on the "population of radicals", the total change of the " $\mathrm{x} / \mathrm{y}$ ratio" after irradiation by a mixed field can be written as follows.

$$
\Delta R G_{c o} D=\Delta R_{\gamma} G_{\gamma} D_{\gamma}+\Delta R_{n} G_{n} D_{n}
$$

In the above equation, " $D$ " is the theoretical absorbed dose. For convenience, the " $D$ " value could be converted to a ${ }^{60} \mathrm{Co}$ equivalent measured dose value: " $d$ ". The LET value of gamma rays is not very sensitive to energy variation and can be regarded as the same value in the case of $G_{\text {co }}$ and $G_{\Gamma}[13]$. However, in the case of neutrons, it requires a different conversion factor.

$$
D=d \quad D_{\gamma}=d_{\gamma} \quad D_{n}=\frac{G_{c o}}{G_{n}} d_{n}
$$

If the above conversions are applied to equation (2), then the equation can be expressed as follows:

$$
\Delta R d=\Delta R_{\gamma} d_{\gamma}+\Delta R_{n}\left(d-d_{\gamma}\right)
$$

For the above equation, the change of the " $\mathrm{x} / \mathrm{y}$ ratio" value by gamma rays irradiation, $\Delta R_{\Gamma}$ and $\Delta R$, could be estimated and calculated for each dosimeter. For $\Delta R_{n}$,

Table 2. Dose and the "x/y ratio" of Alanine Pellets

\begin{tabular}{l|c|c|c|c|c}
\hline Pellet No & 26 & 35 & 47 & 3 & 50 \\
\hline Dose of $1^{\text {st }}$ operation cycle & $748 \mathrm{~Gy}$ & $901 \mathrm{~Gy}$ & $792 \mathrm{~Gy}$ & & $2288 \mathrm{~Gy}$ \\
\hline "x/y ratio" & 0.7567 & 0.7491 & 0.7521 & & 0.7325 \\
\hline Dose of $1^{\text {st }} \& 2^{\text {nd }}$ operation cycles & $1,551 \mathrm{~Gy}$ & $1,814 \mathrm{~Gy}$ & $1,591 \mathrm{~Gy}$ & $908 \mathrm{~Gy}$ & \\
\hline "x/y ratio" & 0.7378 & 0.7236 & 0.7346 & 0.7367 & \\
\hline Dose of $2^{\text {nd }}$ operation cycle & $803 \mathrm{~Gy}$ & $913 \mathrm{~Gy}$ & $799 \mathrm{~Gy}$ & & \\
\hline Change of "x/y ratio" & 0.0189 & 0.0255 & 0.0175 & 0.0333 & 0.0375 \\
\hline$\Delta \mathrm{R}^{*}$ & $2.35 \mathrm{E}-5$ & $2.79 \mathrm{E}-5$ & $2.19 \mathrm{E}-5$ & $3.67 \mathrm{E}-05$ & $1.64 \mathrm{E}-05$ \\
\hline$\Delta \mathrm{R}_{\Gamma}^{* *}$ & $1.66 \mathrm{E}-5$ & $1.61 \mathrm{E}-5$ & $1.70 \mathrm{E}-5$ & $2.40 \mathrm{E}-05$ & $1.21 \mathrm{E}-05$ \\
\hline$\Delta \mathrm{R}_{n}^{* * *}$ & $8.78 \mathrm{E}-05$ & $8.78 \mathrm{E}-05$ & $8.78 \mathrm{E}-05$ & $8.78 \mathrm{E}-05$ & $8.78 \mathrm{E}-05$ \\
\hline $\mathrm{D}_{\Gamma}^{\dagger}$ & $494 \mathrm{~Gy}$ & $526 \mathrm{~Gy}$ & $502 \mathrm{~Gy}$ & $415 \mathrm{~Gy}$ & $1635 \mathrm{~Gy}$ \\
Percentage of radicals & $61.6 \%$ & $57.6 \%$ & $62.9 \%$ & $45.7 \%$ & $71.5 \%$ \\
\hline
\end{tabular}

* change of " $\mathrm{x} / \mathrm{y}$ ratio" at $2^{\text {nd }}$ operation cycle,

** change of " $\mathrm{x} / \mathrm{y}$ ratio" by additive gamma rays irradiation to alanine dosimeters from NPP,

${ }^{* * *}$ estimated value from measured gamma dose and $\Delta \mathrm{R}_{\Gamma}$,

${ }^{\dagger}$ calculated gamma dose using equation (4) 
generally, $\mathrm{D}_{2} \mathrm{O}$ moderated ${ }^{252} \mathrm{Cf}$ neutron source has been used as standard energy spectra to neutron rays for calibration of personal dosimeters in NPPs. However, from previous measurements by institutes, neutron flux and energy spectra in NPPs vary drastically with the measured positions and time. Hence, in the current work, the $\Delta R_{n}$ value was estimated by applying the measured value "d" by e-scan and the gamma dose quantity of position No 35. The gamma dose was based on the gamma dose rate at position No 35 using GM tube detectors at the status of full power operation. All estimation of gamma dose quantity in Table 2 was obtained by application of this $\Delta R_{n}$ value of position No 35 .

\section{CONCLUSION}

Gamma ray radiation in NPP strongly influences the aging of polymer based materials such as power cables and instrumentation cables, although the effect is dependent on the total absorbed dose. Accordingly, estimation of the accumulated gamma dose is important to predict the life expectancy of cables. However, the gamma dose at some positions in the containment building of a NPP is difficult to estimate, because the positions may be exposed to mixed gamma-neutron radiation fields. In addition, for actual measurement during the operation period in a NPP, exposure to an extremely high dose of radiation is inevitable. In this study, the change of the spectrum shape of alanine dosimeters has been used as an indicator to discriminate radiation quality in mixed radiation fields. Estimation of gamma dose for operation cycles was performed using the relation between absolute population size of radicals and change of the " $\mathrm{x} / \mathrm{y}$ ratio" in the spectrum of alanine dosimeters. The estimated portion of free radicals induced by gamma rays was about $42 \sim 72 \%$ of all the radicals in mixed radiation fields. Estimated gamma dose for three positions, No 26,35 , and 47 , with similar radiation environments appeared to be more reliable than gamma dose estimation at positions No 3 and No 50. This estimation method of gamma dose could be applied to the dosimetry results at other NPPs in future studies.

\section{REFERENCES}

[ 1 ] F. Hanisch, P. Maier, S. Okada, H. Onbacherh. "Effects of
Radiation types and dose rates on selected Cable-insulating materials," Raiat. Phys. Chem., Vol 30, No 1, pp 1-9, 1987.

[2 ] IAEA, "Assessment and management of ageing of major nuclear power plant components important to safety: Incontainment instrumentation and control cables", IAEATECDOC-1188, International Atomic Energy Agency, 2000.

[ 3 ] K. Mehta and R. Girzikowsky, "Reference Dosimeter system of the IAEA", Radat. Phys. Chem., Vol 46, No. 4-6, pp 1247-1250, 1995.

[4] J. M. Dolo, N. lecerf and I. Tartes, "Progress in Quality Control of Alanine Dosimeters", Appl. Radiat. Isot., Vol. 47, No.11/12, pp 1165-1168, 1996.

[ 5 ] ASTM E 1607-96, Standard practice for use of the AlanineEPR dosimetry system, American National Standard Institute, 1996.

[6] E. Malinen, M. Z. Heydari, E. Sagstuen and E. O. Hole, "Alanine Radicals, Part 3: Properties of the components contributing to the EPR spectrum of $\mathrm{x}$-irradiatied alanine dosimeters", Radiation Research Vol 159, pp 23-32, 2003.

[7] M. Z. Heydari, E. Malinen, E. O. Hole and E. Sagstuen, "Alanine Radicals, Part 2: The composite Poly crystalline Alanine EPR Spectrum Studied by ENDOR, Thermal Annealing and Spectrum Simulations", J. phys. chem., Vol 106, pp 8971-8977, 2002.

[ 8 ] E. Malinen, E. A. Hult, E. O. Hole and E. Sagstuen, "Alanine Radicals, Part 4: Relative Amounts of Radical Species in Alanine Dosimeters after Exposure to 6-19Mev Electrons and $10 \mathrm{Kv}-15 \mathrm{Mv}$ photons", Radiation Research, Vol 150, pp 149-153, 2003.

[9] B. Ciesieelski, L.Wielopolski, "The effects of boron on the electron paramagnetic resonance spectra of alanine irradiated with thermal neutrons", Radiation research, Vol 144, pp 59-63, 1995.

[10] B. Ciesielski, L.Wielopolski, "The effects of Dose and Radiation Quality on the shape and Power Saturation of the EPR Signal in Alanine", Radiation research, Vol 140, pp 105-111, 1994.

[11] B. Ciesielski, Z. Stuglik, L. Wielopolski and I. Zvara, "The effect of High-Linear Energy Transfer Ions on the Electron Paramagnetic Resonance Signal in Alanine", Radiation Research, Vol 150, pp 469-474, 1998.

[12] V. Nagy, J. M. Puhl, M. F. Desrosiers, "Advancements in accuracy of the alanine dosimetry system: Part 2: The influence of the irradiation temperature", Radiation Physics and Chemistry, Vol 57, pp 1-9, 2000.

[13] A. Cecilla, S. Baccaro, A. Cemmi, V. Colli, G. Rosi and L. Scolari, "Alanine and TLD coupled detectors for fast neutron dose measurements in neutron capture therapy(NCT)", Radiation Protection Dosimetry, Vol 110, Issue 1-4, pp 637-640, 2004. 